\title{
The Pretreatment Neutrophil/ Lymphocyte Ratio Is Associated with All-Cause Mortality in Black and White Patients with Non-metastatic Breast Cancer
}

\author{
Joseph Rimando ${ }^{1 \dagger}$, Jeff Campbell't+, Jae Hee Kim², Shou-Ching Tang ${ }^{1,2}$ and \\ Sangmi Kim ${ }^{1,2 *}$
}

'Section of Hematology/Oncology, Department of Medicine, Medical College of Georgia at Georgia Regents University, Augusta, GA, USA, ${ }^{2}$ Georgia Regents University Cancer Center, Augusta, GA, USA

OPEN ACCESS

Edited by:

Imtiaz Ahmad Siddiqui,

University of Wisconsin-Madison,

USA

Reviewed by:

Mohammad Imran Khan,

University of Wisconsin-Madison,

USA

Chandra Singh,

University of Wisconsin-Madison,

USA

*Correspondence:

Sangmi Kim

sankim@gru.edu

†'Joseph Rimando and Jeff Campbell equally contributed to this work.

Specialty section: This article was submitted to Cancer Epidemiology and Prevention, a section of the journal

Frontiers in Oncology

Received: 19 December 2015

Accepted: 21 March 2016

Published: 31 March 2016

Citation:

Rimando J, Campbell J, Kim JH,

Tang S-C and Kim S (2016) The Pretreatment Neutrophil/Lymphocyte

Ratio Is Associated with All-Cause Mortality in Black and White Patients with Non-metastatic Breast Cancer.

Front. Oncol. 6:81.

doi: 10.3389/fonc.2016.00081
The pretreatment neutrophil/lymphocyte ratio (NLR), derived from differential white blood cell counts, has been previously associated with poor prognosis in breast cancer. Little data exist, however, concerning this association in Black patients, who are known to have lower neutrophil counts than other racial groups. We conducted a retrospective cohort study of 236 Black and 225 non-Hispanic White breast cancer patients treated at a single institution. Neutrophil and lymphocyte counts were obtained from electronic medical records. Univariate and multivariate Cox regression models were used to determine hazard ratios (HRs) and 95\% confidence intervals (95\% Cls) of all-cause mortality and breast cancer-specific mortality in relation to pretreatment NLR. Overall, there were no associations between an elevated pretreatment NLR (NLR $\geq 3.7)$ and all-cause or breast cancer-specific mortality. Among patients without metastasis at the time of diagnosis, an elevated pretreatment NLR was independently associated with all-cause mortality, with a multivariable $\mathrm{HR}$ of 2.31 (95\% Cl: 1.10-4.86). Black patients had significantly lower NLR values than White patients, but there was no evidence suggesting racial heterogeneity of the prognostic utility of NLR. Pretreatment NLR was an independent predictor of all-cause mortality but not breast cancer-specific mortality in non-metastatic breast cancer patients.

Keywords: breast cancer, neutrophil/lymphocyte ratio, Black patients, White patients, all-cause mortality

\section{INTRODUCTION}

Breast cancer is the most common cancer in women and is the second leading cause of cancer death in women in the United States (1). Tumor stage, grade, and tissue markers, such as hormone receptor status and HER2 overexpression, are well-known independent prognostic indicators in breast cancer $(2-5)$. In recent years, increasing data suggest that tumor infiltrating lymphocytes (TILs) may also

Abbreviations: NLR, neutrophil/lymphocyte ratio (the ratio of peripheral blood neutrophils to peripheral blood lymphocytes, obtained from the differential counts of a complete blood count performed on venous blood). 
have prognostic value in breast cancer $(6,7)$. This interaction between the tumor and immune system may also extend beyond the local tissue environment. Indeed, a systemic inflammatory response, determined by elevated circulating levels of C-reactive protein and interleukin-6, has been associated with poor overall and disease-specific survival among breast cancer patients $(8,9)$.

The pretreatment neutrophil/lymphocyte ratio (NLR), derived from differential white blood cell (WBC) counts, is another parameter of systemic inflammation and host immune reaction, which is routinely available in the clinical setting. A few studies have reported that an elevated pretreatment NLR is associated with an increased risk of relapse and worse survival among breast cancer patients (10-19). The pretreatment NLR has been associated with worse prognosis in a variety of different cancers, including colorectal cancer (20), non-small cell lung cancer (21, $22)$, renal cell carcinoma $(23,24)$, gastric cancer $(25,26)$, nasopharyngeal carcinoma $(27,28)$, hepatocellular carcinoma (29), and esophageal cancer (30). Additionally, Chua et al. reported that midtreatment NLR (measured after one cycle of chemotherapy) was associated with improved progression-free survival $(p=0.012)$ in colorectal cancer (31).

Blacks are generally known to have lower neutrophil counts and similar lymphocyte counts compared to their White counterparts (32). However, little data exist concerning the association between the pretreatment NLR and breast cancer prognosis in Black patients. We, therefore, examined whether an elevated pretreatment NLR is associated with all-cause and breast cancerspecific mortality in Black and White patients treated at a single institution.

\section{MATERIALS AND METHODS}

\section{Study Design and Data Source}

Our study was a retrospective review of medical charts of breast cancer patients registered in the Tumor Registry at the Georgia Regents University (GRU) Cancer Center. Out of 1,208 patients registered between April 2001 and August 2013, we identified 464 patients who had differential WBC counts determined prior to initial treatment and excluded 3 patients who were members of racial/ethnic groups other than Black or non-Hispanic White. The total WBC count and the percentage of each neutrophil and lymphocyte were extracted from electronic medical records (EMR) to estimate the absolute neutrophil and lymphocyte counts. The NLR was then calculated by dividing the estimated neutrophil count by the estimated lymphocyte count. Also extracted from the EMR was information on a patient's height and weight at the time of diagnosis or at a proximate time possible to compute body mass index (BMI, kilogram per square meter).

Other major information used in this study was obtained from the hospital tumor registry, which abstracts and codes the data on all patients diagnosed and/or treated for cancer at the GRU Hospital following the state cancer registry standards. This included information on patient characteristics (age at diagnosis, race, primary payer at diagnosis, and tobacco use history), tumor (date of diagnosis, stage at diagnosis, grade, and hormone receptor status), treatment course (surgery, radiation, chemotherapy, and hormone therapy), and follow-up data (date of last contact, date and type of recurrence, and vital status). EMR were further searched among patients expired by the follow-up end date in order to determine if death was attributed to breast cancer. Death was considered a result of breast cancer if (1) "a patient was never been free of breast cancer since diagnosis because of already metastasized diseases and/or treatment failure/refusal" or "a patient was disease-free after initial treatment but had a record of disease recurrence" and (2) medical records within 3 months from the expiration date indicate at least one of the following: widespread metastasis into multiple organs; malignant pleural effusion; significant disease progression and functional decline; or referral to hospice care. The study was approved by the Institutional Review Board of GRU. The informed consent process and the documentation of consent was waived because the study was a retrospective chart review that involved no greater than minimal risk and did not collect protected health information.

\section{Statistical Analysis}

Our primary endpoint was overall survival. Overall survival was calculated from the date of diagnosis to the date of death from any causes (censored) or last follow-up (right-censored). A best cutoff point for NLR was established by examining hazard ratios (HRs), SEs, and $p$-values for various cutoff points obtained from a univariate Cox regression (log-rank test) comparing survival curves of individuals above and below each cutoff point (33, 34). Multivariate Cox regression models were used to determine adjusted HRs after controlling for age, stage, grade, and hormone receptor status (adjustment model 1); adjustment model 1 plus smoking and BMI (adjustment model 2); and all adjustment 1 and 2 factors plus payer type, receipt of chemotherapy, and receipt of radiation therapy (adjustment model 3). A test for linear trend between stage and NLR was conducted by using univariate linear regression. In addition to an analysis, including all patients, we conducted subgroup analyses by race and metastasis status. All analyses were performed in R 3.2.

\section{RESULTS}

This study included 461 (236 Black and 225 White) patients with breast cancer (Table 1). Overall, 122 (65 Black and 57 White) patients died during a median follow-up of 5.1 years [interquartile range (IQR): 5.4]. The median age at diagnosis was 57.8 years (IQR: 19). The median BMI was $29.5 \mathrm{~kg} / \mathrm{m}^{2}$ (IQR: 9.4), and $20 \%$ of patients were current smokers. A majority of patients (90.1\%) had some type of health insurance at the time of diagnosis, and $93 \%$ had a surgery as a part of the treatment compared with 51 and $46 \%$ having radiation and chemotherapy, respectively.

Approximately half of the patients had in situ $(N=81$ or $19 \%)$ or stage I $(N=149$ or $34 \%)$ tumors, followed by $28 \%$ of patients with stage II, $15 \%$ with stage III, and $4 \%$ with stage IV. The proportion of patients with grade I, II, III, and IV tumors was $21,29,49$, and $1 \%$, respectively. The most frequent tumor subtype was the subtype positive for both the estrogen receptor (ER) and progesterone receptor (PR) (62\%). The median counts of WBCs, neutrophils, and lymphocytes were $6.7,4.1$, and 2, respectively. 
TABLE 1 | Patient and clinical characteristics and their relationship with overall survival in a hospital-based retrospective cohort study (2001-2013).

\section{No. (\%) Unadjusted HR $(95 \% \mathrm{Cl})$}

\section{Age}

$<50$

50-64

$\geq 65$

Missing

Race

Black

Non-Hispanic White

\section{BMI}

$<25$

25-29.9

30-34.9

$\geq 35$

Missing

Smoking

Never

Former

Current

Missing

Type of medical insurance

Private/Medicare/uniformed

Medicaid

None

Missing

\section{Stage}

0 and I

II

III

IV

Missing

\section{Grade}

II

III

IV

Missing

Hormone receptor status

$\mathrm{ER}+, \mathrm{PR}+$

$\mathrm{ER}+, \mathrm{PR}-$

ER-, PR+

ER-, PR-

Missing

\section{Type of therapy}

Surgery

Radiation therapy

Chemotherapy

Systemic hormone therapy

Median (interquartile range) Total WBC

Neutrophil count

Lymphocyte count
$129(28.0 \%)$

$186(40.3 \%)$

$146(31.7 \%)$

$1.22(0.79,1.88)$

1. (Ref.)

$1.29(0.84,1.99)$

$236(51.2 \%)$

225 (48.8\%)

$1.08(0.76,1.54)$

1. (Ref.)

108 (25.2\%)

$121(28.2 \%)$

102 (23.8\%)

98 (22.8\%)

32

254 (61.1\%)

$78(18.8 \%)$

$84(20.2 \%)$

45

\section{7 (74.2\%) \\ $73(16.1 \%)$ \\ $44(9.7 \%)$}

\section{$213(52.4 \%)$ \\ 114 (28.1\%) \\ $62(15.3 \%)$ \\ $17(4.2 \%)$}

$84(21.2 \%)$

115 (29.0\%)

193 (48.7\%)

$4(1.0 \%)$

65

$$
\begin{gathered}
244(61.9 \%) \\
46(11.7 \%) \\
9(2.328 \%) \\
95(24.1 \%)
\end{gathered}
$$

67

427/461 (92.6) 232/452 (51.3) 210/458 (45.9) 234/445 (52.6)

$6.7 \pm 2.9$

$4.1 \pm 2.2$

$2.0 \pm 0.8$
1. (Ref.)

$2.22(1.46,3.38)$

$1.64(0.95,2.83)$

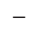

1. (Ref.)

$2.54(1.44,4.50)$

$4.68(2.61,8.38)$

$12.60(6.23,25.30)$

0

1. (Ref.)

$1.67(0.85,3.27)$

$2.18(1.18,4.04)$

1. (Ref.)

$2.18(1.45,3.28)$

$0.13(0.08,0.20)$

$0.63(0.44,0.91)$

$1.77(1.22,2.56)$

$0.54(0.37,0.78)$

$0.98(0.91,1.05)$

$1.02(0.94,1.11)$

$0.74(0.57,0.95)$
As expected, the HRs of all-cause mortality increased in relation to advanced tumor stage [HR for stage IV versus stage 0 or I: $12.60,95 \%$ confidence interval (95\% CI): $6.23-25.30]$, higher grade (HR for grade III/IV versus grade I: 2.18, 95\% CI: 1.18-4.04), and negative hormone receptor status (HR for ERand PR- versus ER+ or PR+: 2.18, 95\% CI: 1.45-3.28). Having Medicaid as a primary payer source and receiving chemotherapy were also associated with elevated risk of all-cause mortality in univariate analyses, but other patient factors, such as age, race, $\mathrm{BMI}$, and smoking history, were not associated with overall survival. No relationship was observed with WBC, neutrophil, or lymphocyte counts.

Black patients had lower NLR values compared with White patients, with a median NLR (IQR) of 1.77 (1.14) for Black patients and 2.28 (1.25) for White patients. This racial difference in NLR remained even when stratified by tumor stage [all $p$-values $<0.001$ except for the stage IV $(p=0.096)$ ] (Figure 1). Interestingly, a positive association between tumor stage and the NLR value was evident only among White patients ( $p$-value for a linear trend $=0.02)$ and was not observed in Black patients ( $p$-value for a linear trend $=0.27$ ). Due to differences in NLR between Black and White patients, we calculated an optimal NLR cutoff point that would significantly predict all-cause mortality in each racial group and in the combined patient population (Figure 2; Table 2). A high HR with the lowest $p$-value was observed at the NLR cutoff value of 3.6 among Black patients and at 3.8 among White patients. However, there was only little difference in the HRs and the associated $p$-values in a range of NLR cutoff values between 3.6 and 3.8 in both racial groups. We thus used an overall optimal cutoff point of 3.7 in subsequent analyses.

We further explored other patient parameters associated with the pretreatment NLR value (Table 3). Patients in the high NLR group defined as NLR $\geq 3.7$ had higher WBC and neutrophil counts but lower lymphocyte counts compared to those in the low NLR group (NLR <3.7) (all $p$-values <0.001). There were more stage IV (17 versus $8 \%$ ) and high-grade tumors (67 versus

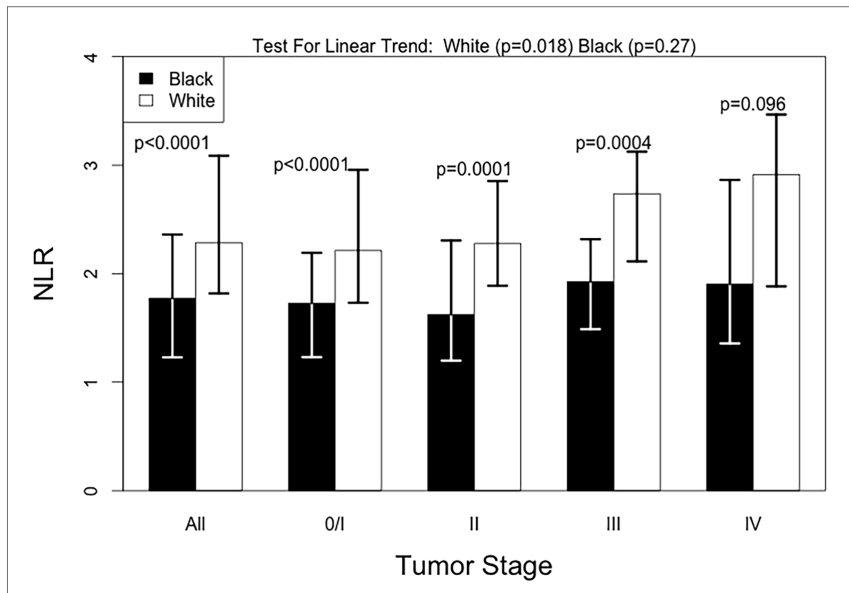

FIGURE 1 | Median NLR levels by tumor stage, according to race. Test for linear trend was done with univariate linear regression and tests by stage were done using the Mann-Whitney test. 

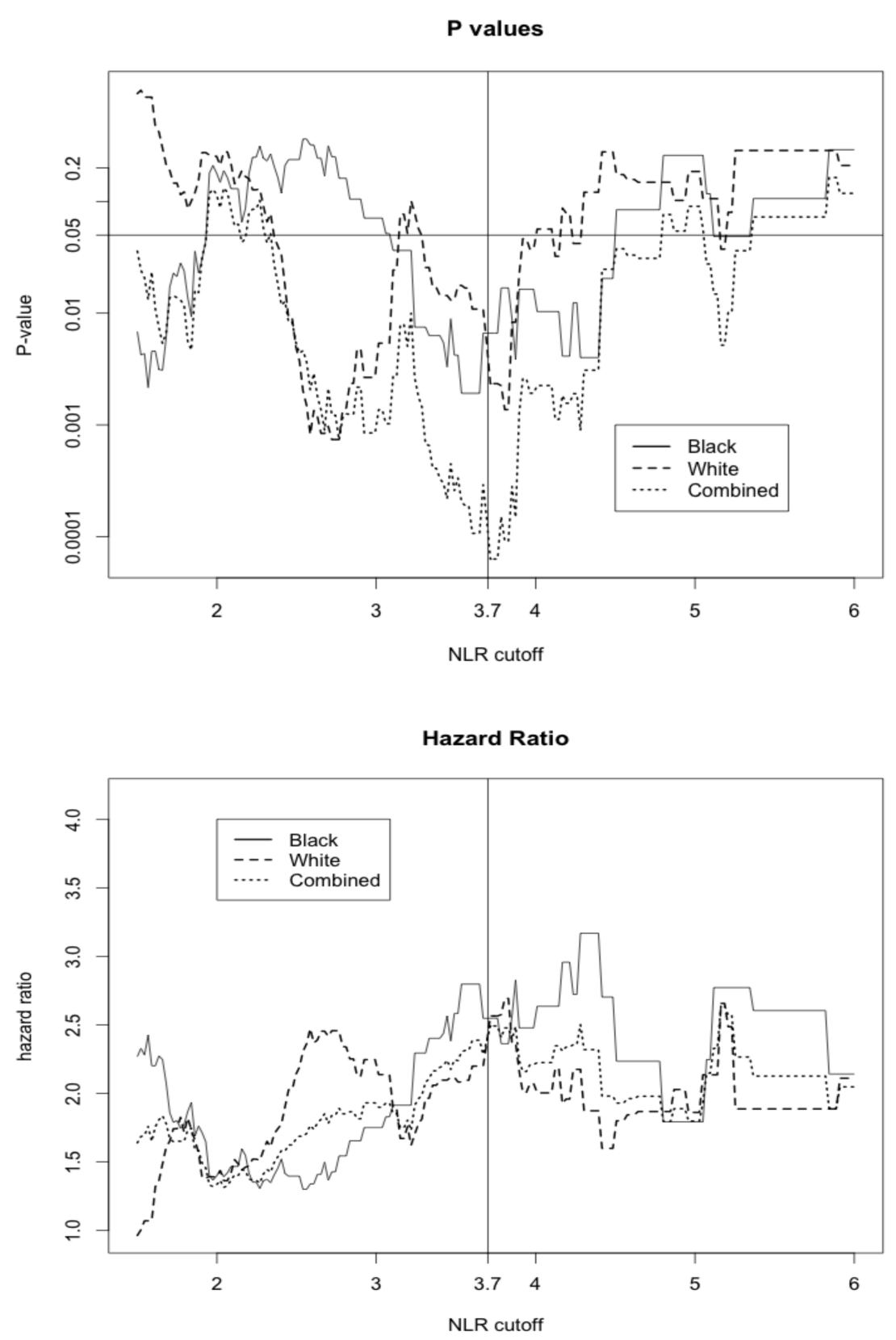

FIGURE 2 | The hazard ratio and $p$-value for all-cause mortality in patients above or equal versus below the corresponding NLR cutoff displayed on $x$-axis.

47\%) in the high NLR group than in the low NLR group, but the differences were not statistically significant. Other patient parameters, such as age, BMI, and smoking status, were also not significantly associated with the NLR groups. Using 3.7 as a cutoff point to define the high NLR group, there were fewer Black patients in the high NLR group compared with White patients, but the difference was only borderline significant (39 versus $61 \%$, $p=0.07)$.

A total of $11 \%$ of breast cancer patients ( $8.5 \%$ of Black and $13.8 \%$ of White) had a pretreatment value of NLR $\geq 3.7$ and was defined to have an elevated pretreatment NLR. In the univariate, unadjusted analysis, high NLR was associated with increased all-cause mortality (HR $=2.38,95 \%$ CI: $1.52-3.72 ; p<0.001$ by the log-rank test) (Table 4; Figure 3). The univariate HR associated with the high NLR was similar between Black $(H R=2.55$, 95\% CI: $1.30-5.00)$ and White (HR $=2.37,95 \%$ CI: $1.29-4.34$ ) patients. After adjustment for age, stage, grade, and combined ER/PR receptor status, however, the high NLR was no longer significantly associated with all-cause mortality $(\mathrm{HR}=1.47,95 \%$ CI: 0.80-2.70). 
TABLE 2 | Race-specific and overall HRs and $p$-values for all-cause mortality across different NLR cutoff points.

\begin{tabular}{|c|c|c|c|c|c|c|c|c|c|}
\hline \multirow[t]{2}{*}{ NLR cutoff } & \multicolumn{3}{|c|}{ Black } & \multicolumn{3}{|c|}{ White } & \multicolumn{3}{|c|}{ All } \\
\hline & HR & SE & $p$ & HR & SE & $p$ & HR & SE & $p$ \\
\hline 2.0 & 1.398 & 0.250 & 0.180 & 1.392 & 0.290 & 0.254 & 1.333 & 0.183 & 0.116 \\
\hline 2.1 & 1.467 & 0.253 & 0.130 & 1.420 & 0.285 & 0.219 & 1.367 & 0.182 & 0.085 \\
\hline 2.2 & 1.500 & 0.257 & 0.115 & 1.471 & 0.275 & 0.160 & 1.416 & 0.181 & 0.055 \\
\hline 2.3 & 1.374 & 0.265 & 0.231 & 1.650 & 0.272 & 0.066 & 1.446 & 0.182 & 0.043 \\
\hline 2.4 & 1.522 & 0.269 & 0.119 & 1.777 & 0.270 & 0.033 & 1.583 & 0.182 & 0.012 \\
\hline 2.5 & 1.397 & 0.283 & 0.237 & 2.137 & 0.271 & 0.005 & 1.666 & 0.184 & 0.005 \\
\hline 2.6 & 1.340 & 0.295 & 0.320 & 2.368 & 0.269 & 0.001 & 1.737 & 0.185 & 0.003 \\
\hline 2.7 & 1.366 & 0.310 & 0.315 & 2.416 & 0.267 & 0.001 & 1.780 & 0.188 & 0.002 \\
\hline 2.8 & 1.544 & 0.310 & 0.161 & 2.338 & 0.266 & 0.001 & 1.850 & 0.191 & 0.001 \\
\hline 2.9 & 1.654 & 0.310 & 0.105 & 2.126 & 0.267 & 0.005 & 1.815 & 0.194 & 0.002 \\
\hline 3.0 & 1.752 & 0.310 & 0.071 & 2.246 & 0.269 & 0.003 & 1.931 & 0.197 & 0.001 \\
\hline 3.1 & 1.914 & 0.310 & 0.036 & 1.886 & 0.282 & 0.024 & 1.854 & 0.206 & 0.003 \\
\hline 3.2 & 1.914 & 0.310 & 0.036 & 1.759 & 0.290 & 0.052 & 1.804 & 0.210 & 0.005 \\
\hline 3.3 & 2.294 & 0.311 & 0.007 & 1.814 & 0.303 & 0.049 & 1.987 & 0.216 & 0.001 \\
\hline 3.4 & 2.439 & 0.321 & 0.005 & 2.097 & 0.303 & 0.014 & 2.204 & 0.218 & $>0.001$ \\
\hline 3.5 & 2.583 & 0.332 & 0.004 & 2.118 & 0.303 & 0.013 & 2.245 & 0.221 & $>0.001$ \\
\hline 3.6 & 2.797 & 0.332 & 0.002 & 2.096 & 0.309 & 0.017 & 2.314 & 0.225 & $>0.001$ \\
\hline 3.7 & 2.548 & 0.344 & 0.007 & 2.369 & 0.309 & 0.005 & 2.381 & 0.228 & $>0.001$ \\
\hline 3.8 & 2.363 & 0.360 & 0.017 & 2.693 & 0.309 & 0.001 & 2.478 & 0.232 & $>0.001$ \\
\hline 3.9 & 2.478 & 0.378 & 0.016 & 2.162 & 0.337 & 0.022 & 2.238 & 0.250 & 0.001 \\
\hline 4.0 & 2.478 & 0.378 & 0.016 & 2.072 & 0.350 & 0.037 & 2.210 & 0.256 & 0.002 \\
\hline 4.1 & 2.636 & 0.378 & 0.010 & 2.004 & 0.365 & 0.057 & 2.225 & 0.262 & 0.002 \\
\hline 4.2 & 2.957 & 0.378 & 0.004 & 1.959 & 0.383 & 0.079 & 2.338 & 0.269 & 0.002 \\
\hline 4.3 & 3.168 & 0.401 & 0.004 & 1.873 & 0.405 & 0.121 & 2.320 & 0.284 & 0.003 \\
\hline 4.4 & 3.168 & 0.401 & 0.004 & 1.873 & 0.405 & 0.121 & 2.320 & 0.284 & 0.003 \\
\hline 4.5 & 2.703 & 0.429 & 0.020 & 1.600 & 0.433 & 0.278 & 1.981 & 0.304 & 0.020 \\
\hline
\end{tabular}

Compared with patients without metastasis, patients with metastatic breast cancer are more likely to have overtly altered inflammatory states and tumor responses $(35,36)$ and shorter survival largely attributed to breast cancer. We, thus, analyzed the relationship between NLR and all-cause mortality, after excluding stage IV breast cancer patients (Table 3). The unadjusted HRs associated with high NLR were 2.43 (95\% CI: 1.36-4.35) in all patients and 2.78 (95\% CI: 1.16-6.64) and 2.28 (95\% CI: 1.03-5.03) among Black and White patients, respectively. The high NLR remained a significant independent prognostic factor for overall survival among non-metastatic breast cancer patients after adjustment for the clinical factors of age, stage, grade, and combined ER/PR receptor status ( $\mathrm{HR}=2.12,95 \%$ CI: 1.05-4.29) and additional adjustment for the patient factors of BMI and smoking history and the treatment factors of payer category, chemotherapy regimen, and radiation therapy regimen ( $\mathrm{HR}=2.27,95 \% \mathrm{CI}: 1.02-5.08)$.

Out of 122 patients who died during the study follow-up, 54 (10 in high NLR group and 44 in low NLR group) deaths were determined attributable to breast cancer. High NLR was significantly associated with breast cancer-specific mortality (unadjusted HR = 2.16, $95 \mathrm{CI}$ : 1.09-4.30) but the association no longer remained after adjustment for clinical factors (adjusted $\mathrm{HR}=1.78$, 95\% CI: 0.71-4.42) (Table 5). Race and tumor metastasis status did not influence the relationship between the NLR and breast cancer-specific mortality.

\section{DISCUSSION}

In a cohort of 461 Black and White patients with breast cancer, elevated pretreatment NLR, defined as 3.7 or higher, was not significantly associated with all-cause or breast cancer-specific mortality after considering patient and clinical factors. By contrast, elevation in pretreatment NLR was an independent predictor of all-cause mortality among breast cancer patients without metastasis at the time of diagnosis.

Some previous studies have reported that NLR is associated with breast cancer-specific mortality. Forget et al. found decreased recurrence-free survival in breast cancer patients with elevated pretreatment NLR, but multivariate analyses were not conducted in that study due to small sample size (15). More recently, KrennPilko et al. reported that NLR $>3$ is an independent predictor of poor disease-free survival but not of overall survival in 762 European female breast cancer patients (37). Pretreatment NLR $\geq 2.5$ was independently associated with breast cancer-specific mortality in two studies of Asian patients with stage I-III breast cancer $(13,14)$. One of the studies also showed that elevated NLR was associated with worse disease-specific survival particularly among patients with the luminal A subtype breast cancer (13). On the other hand, a pretreatment NLR $\geq 2.5$ was not associated with the 21-gene recurrence score in a population of $242 \mathrm{ER}+$ breast cancer patients (38). A significant association of pretreatment NLR with both overall and disease-free survival has been 
reported in studies based on triple-negative breast cancer patients $(19,39)$. In a population of 187 HER2-positive breast cancer patients treated with adjuvant trastuzumab in medical centers in Turkey, Ulas et al. reported that patients with a pretreatment NLR

TABLE 3 | Comparison of host and tumor characteristics between patients with high NLR $(\geq 3.7)$ versus low NLR $(<3.7)$ in a hospital-based retrospective cohort study (2001-2013).

\begin{tabular}{|c|c|c|c|}
\hline & NLR $<3.7$ & $N L R \geq 3.7$ & $p$-Value \\
\hline Total no. & 409 & 52 & \\
\hline \multicolumn{4}{|l|}{ Median \pm IQR } \\
\hline White blood cells & $6.60 \pm 2.70$ & $7.75 \pm 3.42$ & $<0.0001$ \\
\hline Neutrophils & $3.78 \pm 2.06$ & $5.82 \pm 2.82$ & $<0.0001$ \\
\hline Lymphocytes & $2.08 \pm 0.80$ & $1.22 \pm 0.666$ & $<0.0001$ \\
\hline Monocytes & $0.47 \pm 0.23$ & $0.48 \pm 0.32$ & 0.75 \\
\hline Age & $57.60 \pm 19.40$ & $57.40 \pm 15.30$ & 0.819 \\
\hline BMl & $29.70 \pm 9.49$ & $27.5 \pm 9.72$ & 0.819 \\
\hline \multicolumn{4}{|l|}{ No. (\%) } \\
\hline Race & & & 0.071 \\
\hline Black & $216(52.8)$ & $20(38.5)$ & \\
\hline White & $193(47.2)$ & $32(61.5)$ & \\
\hline Stage & & & 0.198 \\
\hline I & $192(50.7)$ & $20(41.7)$ & \\
\hline ॥ & $104(27.4)$ & $12(25.0)$ & \\
\hline III & $53(14.0)$ & $8(16.7)$ & \\
\hline IV & $30(7.9)$ & $8(16.7)$ & \\
\hline Grade & & & 0.1 \\
\hline I & 78 (22.3) & $6(14.3)$ & \\
\hline ॥ & $107(30.6)$ & $8(19.0)$ & \\
\hline III/IV & $165(47.1)$ & $28(66.7)$ & \\
\hline Smoking & & & 0.85 \\
\hline Never & $227(61.5)$ & 27 (57.4) & \\
\hline Former & 68 (18.4) & $10(21.3)$ & \\
\hline Current & $74(20.1)$ & $10(21.3)$ & \\
\hline Payer & & & 0.833 \\
\hline Private/Medicare/uniformed & $301(74.5)$ & $36(72.0)$ & \\
\hline Medicaid/none & $103(25.5)$ & $14(28.0)$ & \\
\hline
\end{tabular}

$>2.38$ had shorter disease-free survival, but their findings were non-significant $(p=0.45)(40)$.

Several studies also point to the association of pretreatment NLR with overall survival in breast cancer patients. A meta-analysis of five studies published in 2014 has summarized that elevated pretreatment NLR, with the cutoff values ranged between 2.0 and 4.0 , was associated with a significant increase in all-cause mortality ( $\mathrm{HR}=2.3,95 \% \mathrm{CI}: 1.1-4.8)$ but to a lesser extent with disease-free survival (HR = 1.4, 95\% CI: 0.9-2.1) (17). Subgroup analyses also indicated that the significant association between pretreatment NLR and prognostic utility of NLR is more evident among White versus Asian patients (17). Consistent with this notion, a recent large study based on patients of different Asian ethnic groups reported a significant, but modest, association between high pretreatment NLR and overall death among breast cancer patients (18). However, to the best of our knowledge, there has been no research that included adequate number of Black patients.

Black individuals have lower WBC and absolute neutrophil counts compared with White individuals (32) primarily due to genetic deletion of the Duffy antigen receptor for chemokines that has been hypothesized to affect the number of circulating neutrophils $(41,42)$. Consistent with this understanding, Black patients in our dataset had lower NLR values than White patients, even after stratifying patients by tumor stage. This trend led us to determine different optimal NLR cutoff points for Black and White patients separately. Reflecting an overall lower NLR value in Black patients, a slightly lower NLR cutoff was selected for Black patients compared with White patients, but the difference was not large enough to warrant race-specific cutoff points. We also found that the association between pretreatment NLR value and tumor stage was less evident among Black patients. However, the association between NLR and all-cause and breast cancerspecific mortality remained similar with comparable estimates using either race-specific or a unified cutoff point value, and there was no evidence suggesting racial heterogeneity of the prognostic utility of NLR.

Inflammation and host immune response can be a critical factor for breast cancer prognosis by altering the tumor microenvironment and therapeutic efficacy (43). Peripheral immune

TABLE 4 | All-cause mortality in relation to high NLR ( $\geq 3.7)$ versus low NLR (<3.7).

\begin{tabular}{|c|c|c|c|c|c|c|c|c|c|}
\hline & \multicolumn{3}{|c|}{ Black } & \multicolumn{3}{|c|}{ White } & \multicolumn{3}{|c|}{ Combined } \\
\hline & Deaths & HR & $95 \% \mathrm{Cl}$ & Deaths & HR & $95 \% \mathrm{Cl}$ & Deaths & HR & $95 \% \mathrm{Cl}$ \\
\hline \multicolumn{10}{|l|}{ All stages } \\
\hline Unadjusted & 65 & 2.55 & $(1.30,5.00)$ & 57 & 2.37 & $(1.29,4.34)$ & 122 & 2.38 & $(1.52,3.72)$ \\
\hline Adjustment model 1 & 44 & 1.86 & $(0.78,4.46)$ & 32 & 1.04 & $(0.39,2.76)$ & 76 & 1.47 & $(0.80,2.70)$ \\
\hline Adjustment model 2 & 35 & 1.82 & $(0.66,5.05)$ & 25 & 1.76 & $(0.56,5.57)$ & 60 & 1.52 & $(0.79,2.91)$ \\
\hline Adjustment model 3 & 35 & 1.3 & $(0.43,3.91)$ & 25 & 1.49 & $(0.44,5.07)$ & 60 & 1.47 & $(0.73,2.96)$ \\
\hline \multicolumn{10}{|l|}{ Excluding stage IV } \\
\hline Unadjusted & 40 & 2.78 & $(1.16,6.64)$ & 35 & 2.28 & $(1.03,5.03)$ & 75 & 2.43 & $(1.36,4.35)$ \\
\hline Adjustment model 1 & 34 & 2.53 & $(0.97,6.62)$ & 23 & 1.68 & $(0.51,5.53)$ & 57 & 2.12 & $(1.05,4.29)$ \\
\hline Adjustment model 2 & 25 & 3.68 & $(1.04,13.00)$ & 18 & 3.17 & $(0.82,12.3)$ & 43 & 2.44 & $(1.12,5.31)$ \\
\hline Adjustment model 3 & 25 & 2.88 & $(0.72,11.60)$ & 18 & 2.86 & $(0.69,11.8)$ & 43 & 2.27 & $(1.02,5.08)$ \\
\hline
\end{tabular}

Adjustment model 1 controlled for stage (O/I, II, III, and IV), grade (I, II, and III/IV), hormone receptor status (ER+ or PR+, ER-, and PR-), and age (continuous). In addition to the variables in adjustment model 1, adjustment model 2 controlled for smoking (former and current versus never) and BMI (<25, 25-2.9, 30-34.9, and 35+). In addition to the variables in adjustment model 2, adjustment model 3 controlled for payer type (private, Medicare, or Uniform, and None or Medicaid), receipt of chemotherapy, and receipt of radiation therapy. 

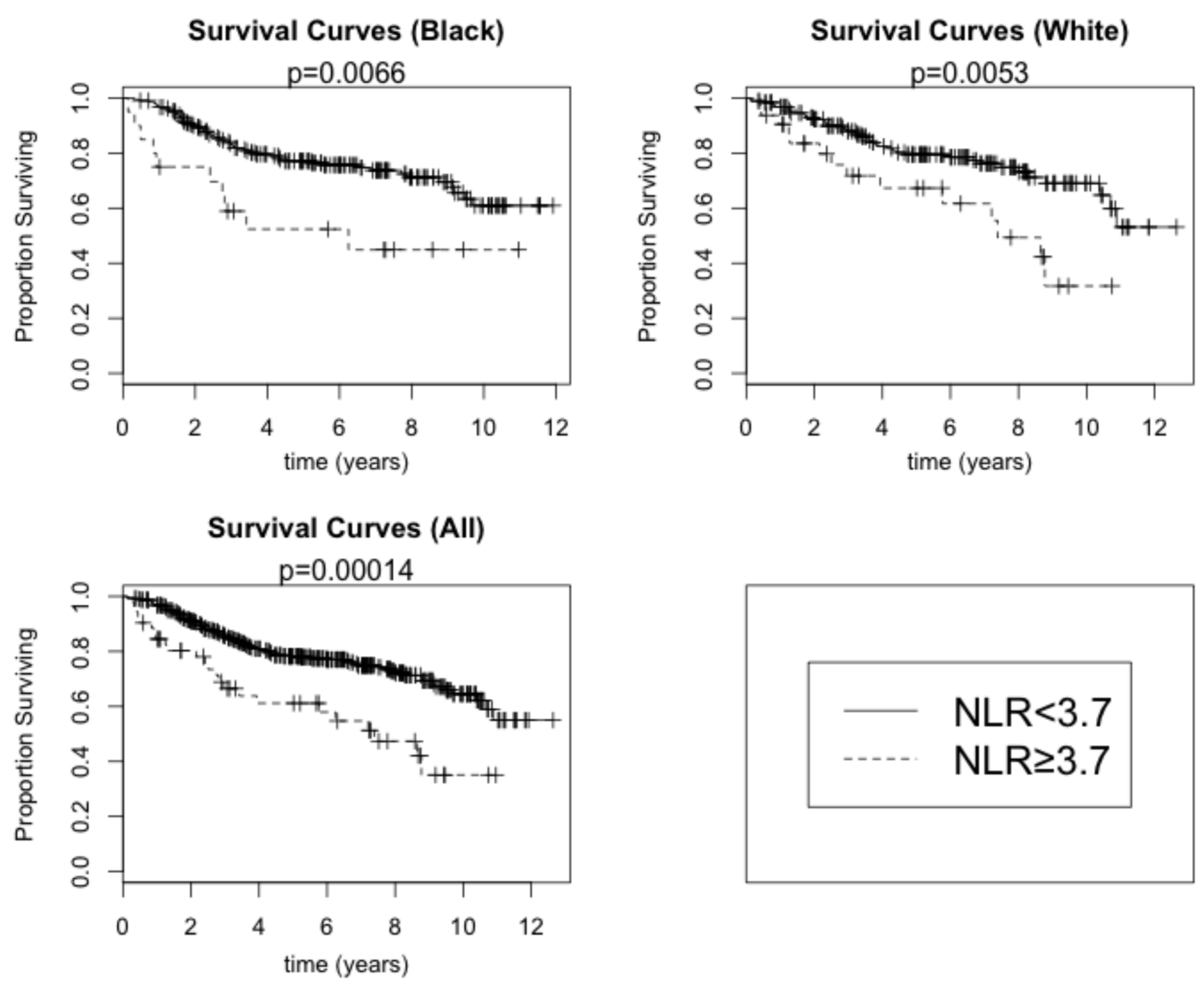

FIGURE 3 | Overall survival by high NLR ( $\geq 3.7)$ versus low NLR (<3.7) among Black and White breast cancer patients. $p$-Values are for the log-rank test.

TABLE 5 | Breast cancer-specific mortality in relation to high NLR ( $\geq 3.7)$ versus low NLR $(<3.7)$.

\begin{tabular}{|c|c|c|c|c|c|c|c|c|c|}
\hline & \multicolumn{3}{|c|}{ Black } & \multicolumn{3}{|c|}{ White } & \multicolumn{3}{|c|}{ Combined } \\
\hline & Deaths & HR & $95 \% \mathrm{Cl}$ & Deaths & HR & $95 \% \mathrm{Cl}$ & Deaths & HR & $95 \% \mathrm{Cl}$ \\
\hline \multicolumn{10}{|l|}{ All stages } \\
\hline Unadjusted & 33 & 2.47 & $(0.95,6.39)$ & 21 & 2.15 & $(0.79,5.86)$ & 54 & 2.16 & $(1.09,4.3)$ \\
\hline Adjustment model 1 & 24 & 1.08 & $(0.30,3.94)$ & 10 & 1.41 & $(0.25,7.96)$ & 34 & 1.78 & $(0.71,4.42)$ \\
\hline Adjustment model 2 & 22 & 0.57 & $(0.12,2.73)$ & 8 & 4.74 & $(0.23,99.30)$ & 30 & 1.27 & $(0.46,3.45)$ \\
\hline Adjustment model 3 & 22 & 0.59 & $(0.10,3.45)$ & 8 & a & a & 30 & 1.46 & $(0.46,4.62)$ \\
\hline \multicolumn{10}{|l|}{ Excluding stage IV } \\
\hline Unadjusted & 17 & 3.31 & $(0.95,11.60)$ & 6 & 1.51 & $(0.18,12.90)$ & 23 & 2.22 & $(0.75,6.53)$ \\
\hline Adjustment model 1 & 16 & 2.73 & $(0.75,10.00)$ & 3 & a & a & 19 & 2.71 & $(0.86,8.56)$ \\
\hline Adjustment model 2 & 14 & 1.40 & $(0.26,7.51)$ & 2 & a & a & 16 & 1.52 & $(0.40,5.85)$ \\
\hline Adjustment model 3 & 14 & 1.06 & $(0.16,7.16)$ & 2 & a & a & 16 & 1.69 & $(0.38,7.55)$ \\
\hline
\end{tabular}

aModel did not converge. Adjustment model 1 controlled for stage (O/I, II, III, and IV), grade (I, II, and III/I), hormone receptor status (ER+ or PR+, ER-, and PR-), and age (continuous). In addition to the variables in adjustment model 1, adjustment model 2 controlled for smoking (former and current versus never) and BMI (<25, 25-2.9, 30-34.9, and 35+). In addition to the variables in adjustment model 2, adjustment model 3 controlled for payer type (private, Medicare, or Uniform, and None or Medicaid), receipt of chemotherapy, and receipt of radiation therapy.

parameters, such as NLR studied in the present study, are a plausible and practically attractive marker of systemic inflammatory responses (44) and may reflect tumor-specific immune response. In a recent study of non-small cell lung cancer patients, the high NLR was correlated with TILs and independently associated with the prognosis (45). However, NLR largely represents a nonspecific indicator of an immune state (46). For example, an elevated NLR has been linked to worse outcomes in various diseases across several different cancer types and those outside of cancer, including coronary artery disease and heart failure (47-49). In a 
large cohort study that included both patients with and without cancer, high neutrophil and low lymphocyte counts were associated with an increase in all-cause mortality and cardiovascular mortality as well as cancer-specific mortality (48). It is known that women, especially elderly women, diagnosed with early stage breast cancers are more likely to die because of cardiovascular diseases than from breast cancer (50). We were unable to estimate cardiovascular disease-specific mortality or its contribution to the observed association between NLR and all-cause mortality in our cohort. However, these findings, including our study, emphasize the pervasive effect that inflammation has on several aspects of health in several different organ systems and processes. It should be also noted that although this study focused on NLR, the prognostic implication of inflammation could not be fully captured, using this single marker of inflammation. However, combined with other markers of local and systemic inflammation, NLR, a low-cost, reliable marker of inflammation may have potential utility in predicting short- and long-term prognosis and risk stratification for anti-inflammatory or immune therapy for cancer patients in the future.

Several strengths and limitations exist within the study. In terms of strengths, this study is one of the first to determine the association of pretreatment NLR to both all-cause and breast cancer-specific mortality in a group of Black and White patients. Nearly, equal numbers of Black and White patients were included, allowing us to explore a meaningful cutoff point in both racial groups. We used a cutoff point determination method for survival analysis to select a cutoff point that maximizes the relation with the HRs and SEs in Black and White patients $(33,34)$. Because the distribution of NLR varies in the two racial groups and the biological meaning in terms of breast cancer prognosis is unknown, we believe this method was well suited to the nature of our data and the specific purpose of our study to examine the association between NLR and prognosis in Black and White patients. However, the prognostic value of our chosen cutoff point can be only determined after validation in independent datasets.

In terms of weaknesses, our study relied on medical records to identify breast cancer-specific death based on a priori criteria. Our data had comparable numbers of breast cancer-specific deaths by stage to those reported in a previous national death

\section{REFERENCES}

1. Siegel RL, Miller KD, Jemal A. Cancer statistics, 2015. CA Cancer J Clin (2015) 65(1):5-29. doi:10.3322/caac.21254

2. American Joint Committee on Cancer. AJCC Cancer Staging Handbook: From the AJCC Cancer Staging Manual. New York: Springer (2002).

3. Elston CW, Ellis IO. Pathological prognostic factors in breast cancer. I. The value of histological grade in breast cancer: experience from a large study with long-term follow-up. Histopathology (1991) 41(3a):403-10. doi:10.111 1/j.1365-2559.1991.tb00229.x

4. Osborne CK, Yochmowitz MG, Knight WA, McGuire WL. The value of estrogen and progesterone receptors in the treatment of breast cancer. Cancer (1980) 46(S12):2884-8. doi:10.1002/1097-0142(19801215)46:12+<2884::AIDCNCR2820461429>3.0.CO;2-U

5. Slamon DJ, Clark GM, Wong SG, Levin WJ, Ullrich A, McGuire WL. Human breast cancer: correlation of relapse and survival with amplification of the HER-2/neu oncogene. Science (1987) 235(21):0-5. doi:10.1126/ science.3798106 registry-based study (51), but some unquantifiable level of classification bias is unavoidable. The nature of a retrospective chart review created additional limitations to our study. First, while we began with a cohort of 461 patients, we lost several patients in subsequent models adjusting for potential confounding factors due to incomplete patient records. Thus, while the adjustment models became subsequently more specific in their analysis, they may have lost statistical significance due to decreasing numbers of patients included in the model. Second, we lacked patient data on cardiometabolic health status and cardiovascular health risk factors. As NLR has been previously shown to be associated with increased prevalence of coronary artery disease and cardiovascular mortality $(48,49,52-55)$, the relationship between NLR and cardiometabolic status in our patient cohort could have further weakened the specificity of NLR for breast cancer-specific mortality. Finally, the present study conducted subgroup analyses by race and metastasis status at diagnosis. Although the analyses were planned and guided by biological plausibility, the resulting increased number of testing will raise the probability of false positive findings.

The results of the present retrospective cohort of 461 Black and White breast cancer patients showed that elevated NLR defined as an NLR of 3.7 or higher is an independent predictor of all-cause mortality in non-metastatic breast cancer patients. Further studies, preferably in a patient population with available cardiovascular health data, are needed to clarify the appropriate clinical context to use NLR as a prognostic factor.

\section{AUTHOR CONTRIBUTIONS}

JR, JC, JK, S-CT, and SK designed the study presented in this manuscript. JR and SK collected data from the electronic health records for this study. JC and JK performed the statistical analyses for this study. JR, JC, and SK wrote the manuscript and created the tables and figures with input from JK and S-CT.

\section{FUNDING}

This work was supported by Georgia Regents University Research Start-Up Fund.

6. Baker K, Lachapelle J, Zlobec I, Bismar TA, Terracciano L, Foulkes WD. Prognostic significance of CD8+ T lymphocytes in breast cancer depends upon both oestrogen receptor status and histological grade. Histopathology (2011) 58(7):1107-16. doi:10.1111/j.1365-2559.2011. 03846.x

7. Ali HR, Provenzano E, Dawson SJ, Blows FM, Liu B, Shah M, et al. Association between CD8+ T-cell infiltration and breast cancer survival in 12,439 patients. Ann Oncol (2014) 25(8):1536-43. doi:10.1093/annonc/mdu191

8. Allin KH, Nordestgaard BG, Flyger H, Bojesen SE. Elevated pre-treatment levels of plasma C-reactive protein are associated with poor prognosis after breast cancer: a cohort study. Breast Cancer Res (2011) 13(3):R55. doi:10.1186/ bcr2891

9. Goldberg JE, Schwertfeger KL. Proinflammatory cytokines in breast cancer: mechanisms of action and potential targets for therapeutics. Curr Drug Targets (2010) 11(9):1133-46. doi:10.2174/138945010792006799

10. Wang S, Zhang Z, Fang F, Gao X, Sun W, Liu H. The neutrophil/lymphocyte ratio is an independent prognostic indicator in patients with bone metastasis. Oncol Lett (2011) 2:735-40. doi:10.3892/ol.2011.304 
11. Azab B, Bhatt VR, Phookan J, Murukutla S, Kohn N, Terjanian T, et al. Usefulness of the neutrophil-to-lymphocyte ratio in predicting short- and long-term mortality in breast cancer patients. Ann Surg Oncol (2012) 19(1):217-24. doi:10.1245/s10434-011-1814-0

12. Azab B, Shah N, Radbel J, Tan P, Bhatt V, Vonfrolio S, et al. Pretreatment neutrophil/lymphocyte ratio is superior to platelet/lymphocyte ratio as a predictor of long-term mortality in breast cancer patients. Med Oncol (2013) 30(1):432. doi:10.1007/s12032-012-0432-4

13. Noh H, Eomm M, Han A. Usefulness of pretreatment neutrophil to lymphocyte ratio in predicting disease-specific survival in breast cancer patients. J Breast Cancer (2013) 16(1):55-9. doi:10.4048/jbc.2013.16.1.55

14. Nakano K, Hosoda M, Yamamoto M, Yamashita H. Prognostic significance of pre-treatment neutrophil: lymphocyte ratio in Japanese patients with breast cancer. Anticancer Res (2014) 34:3819-24.

15. Forget P, Machiels J-P, Coulie PG, Berliere M, Poncelet AJ, Tombal B, et al. Neutrophil:lymphocyte ratio and intraoperative use of ketorolac or diclofenac are prognostic factors in different cohorts of patients undergoing breast, lung, and kidney cancer surgery. Ann Surg Oncol (2013) 20(Suppl 3):S650-60. doi:10.1245/s10434-013-3136-X

16. Eryilmaz MK, Mutlu H, Salim DK, Yalcin F, Tural D, Coskun HS. The neutrophil to lymphocyte ratio has a high negative predictive value for pathologic complete response in locally advanced breast cancer patients receiving neoadjuvant chemotherapy. Asian Pac J Cancer Prev (2014) 15:7737-40. doi:10.7314/APJCP.2014.15.18.7737

17. Chen J, Deng Q, Pan Y, He B, Ying H, Sun H, et al. Prognostic value of neutrophil-to-lymphocyte ratio in breast cancer. FEBS Open Bio (2015) 5:502-7. doi:10.1016/j.fob.2015.05.003

18. Koh C-H, Bhoo-Pathy N, Ng K-L, Jabir RS, Tan G-H, See M-H, et al. Utility of pre-treatment neutrophil-lymphocyte ratio and platelet-lymphocyte ratio as prognostic factors in breast cancer. Br J Cancer (2015) 113(1):150-8. doi:10.1038/bjc.2015.183

19. Pistelli M, De Lisa M, Ballatore Z, Caramanti M, Pagliacci A, Battelli N, et al. Pre-treatment neutrophil to lymphocyte ratio may be a useful tool in predicting survival in early triple negative breast cancer patients. BMC Cancer (2015) 15(1):195. doi:10.1186/s12885-015-1204-2

20. Kaneko M, Nozawa H, Sasaki K, Hongo K, Hiyoshi M, Tada N, et al. Elevated neutrophil to lymphocyte ratio predicts poor prognosis in advanced colorectal cancer patients receiving oxaliplatin-based chemotherapy. Oncology (2012) 82(5):261-8. doi:10.1159/000337228

21. Sarraf KM, Belcher E, Raevsky E, Nicholson AG, Goldstraw P, Lim E. Neutrophil/lymphocyte ratio and its association with survival after complete resection in non-small cell lung cancer. J Thorac Cardiovasc Surg (2009) 137(2):425-8. doi:10.1016/j.jtcvs.2008.05.046

22. Cedrés S, Torrejon D, Martínez A, Martinez P, Navarro A, Zamora E, et al. Neutrophil to lymphocyte ratio (NLR) as an indicator of poor prognosis in stage IV non-small cell lung cancer. Clin Transl Oncol (2012) 14(11):864-9. doi:10.1007/s12094-012-0872-5

23. Ohno Y, Nakashima J, Ohori M, Hatano T, Tachibana M. Pretreatment neutrophil-to-lymphocyte ratio as an independent predictor of recurrence in patients with nonmetastatic renal cell carcinoma. J Urol (2010) 184(3):873-8. doi:10.1016/j.juro.2010.05.028

24. Keizman D, Ish-Shalom M, Huang P, Eisenberger MA, Pili R, Hammers H, et al. The association of pre-treatment neutrophil to lymphocyte ratio with response rate, progression free survival and overall survival of patients treated with sunitinib for metastatic renal cell carcinoma. Eur J Cancer (2012) 48(2):202-8. doi:10.1016/j.ejca.2011.09.001

25. Shimada H, Takiguchi N, Kainuma O, Soda H, Ikeda A, Cho A, et al. High preoperative neutrophil-lymphocyte ratio predicts poor survival in patients with gastric cancer. Gastric Cancer (2010) 13(3):170-6. doi:10.1007/ s10120-010-0554-3

26. Jung MR, Park YK, Jeong O, Seon JW, Ryu SY, Kim DY, et al. Elevated preoperative neutrophil to lymphocyte ratio predicts poor survival following resection in late stage gastric cancer. J Surg Oncol (2011) 104(5):504-10. doi:10.1002/ jso. 21986

27. An X, Ding PR, Wang FH, Jiang WQ, Li YH. Elevated neutrophil to lymphocyte ratio predicts poor prognosis in nasopharyngeal carcinoma. Tumour Biol (2011) 32(2):317-24. doi:10.1007/s13277-010-0124-7

28. He J, Shen G, Ren A, Qin H, Cui C, Zhang Y, et al. Pretreatment levels of peripheral neutrophils and lymphocytes as independent prognostic factors in patients with nasopharyngeal carcinoma. Head Neck (2012) 34(12):1769-76. doi:10.1002/hed.22008

29. Gomez D, Farid S, Malik HZ, Young L, Toogood GJ, Lodge JP, et al. Preoperative neutrophil-to-lymphocyte ratio as a prognostic predictor after curative resection for hepatocellular carcinoma. World J Surg (2008) 32(8):1757-62. doi:10.1007/s00268-008-9552-6

30. Sharaiha RZ, Halazun KJ, Mirza F, Port JL, Lee PC, Neugut AI, et al. Elevated preoperative neutrophil: lymphocyte ratio as a predictor of postoperative disease recurrence in esophageal cancer. Ann Surg Oncol (2011) 18(12):3362-9. doi:10.1245/s10434-011-1754-8

31. Chua W, Charles KA, Baracos VE, Clarke SJ. Neutrophil/lymphocyte ratio predicts chemotherapy outcomes in patients with advanced colorectal cancer. Br J Cancer (2011) 104(8):1288-95. doi:10.1038/bjc.2011.100

32. Hsieh M, Everhart J, Byrd-Holt D, Tisdale J, Rodgers G. Prevalence of neutrophenia in the U.S. population: age, sex, smoking status, and ethnic differences. Ann Intern Med (2007) 146(7):486-92. doi:10.7326/0003-4819-146-7-200704030-00004

33. Mandrekar JN, Mandrekar SJ, Cha SS. Cutpoint determination methods in survival analysis using SAS. Proceedings of the 28th SAS Users Group International Conference (SUGI). Seattle (2003).

34. Heiss MM, Ströhlein MA, Bokemeyer C, Arnold D, Parsons SL, Seimetz D, et al. The role of relative lymphocyte count as a biomarker for the effect of catumaxomab on survival in malignant ascites patients: results from a phase II/III study. Clin Cancer Res (2014) 20(12):3348-57. doi:10.1158/1078-0432. CCR-13-2351

35. Coussens LM, Werb Z. Inflammation and cancer. Nature (2002) 420(6917):860-7. doi:10.1038/nature01322

36. Joyce J, Pollard JW. Microenvironmental regulation of metastasis. Nat Rev Cancer (2009) 9(4):239-52. doi:10.1038/nrc2618

37. Krenn-Pilko S, Langsenlehner U, Stojakovic T, Pichler M, Gerger A, Kapp K, et al. The elevated preoperative derived neutrophil-to-lymphocyte ratio predicts poor clinical outcome in breast cancer patients. Tumour Biol (2015):1-8. doi:10.1007/s13277-015-3805-4

38. Grenader T, Plotkin Y, Geffen DB. The preoperative neutrophil/lymphocyte ratio does not correlate with the 21-gene recurrence score in estrogen receptor-positive breast cancer patients. Oncol Res Treat (2015) 38(1-2):24-7. doi:10.1159/000370341

39. Hong J, Mao Y, Chen X, Zhu L, He J, Chen W, et al. Elevated preoperative neutrophil-to-lymphocyte ratio predicts poor disease-free survival in Chinese women with breast cancer. Tumour Biol (2015).

40. Ulas A, Avci N, Kos T, Cubukcu E, Olmez OF, Degirmenci M. Are neutrophil/ lymphocyte ratio and platelet/lymphocyte ratio associated with prognosis in patients with HER2- positive early breast cancer receiving adjuvant trastuzumab? J BUON (2015) 20(3):714-22.

41. Bonecchi R, Borroni EM, Savino B, Buracchi C, Mantovani A, Locati M. Non-signaling chemokine receptors: mechanism of action and role in vivo. J Neuroimmunol (2008) 198(1-2):14-9. doi:10.1016/j. jneuroim.2008.04.018

42. Reich D, Nalls MA, Kao WHL, Akylbekova EL, Tandon A, Patterson N, et al. Reduced neutrophil count in people of African descent is due to a regulatory variant in the Duffy antigen receptor for chemokines gene. PLoS Genet (2009) 5(1):e1000360. doi:10.1371/journal.pgen.1000360

43. Jiang X, Shapiro DJ. The immune system and inflammation in breast cancer. Mol Cell Endocrinol (2014) 382(1):673-82. doi:10.1016/j.mce.2013.06.003

44. Characiejus D, Jacobs JJL, Pašukonienè V, Kazlauskaitė N, Danilevičiūtè V, Mauricas M, et al. Prediction of response in cancer immunotherapy. Anticancer Res (2011) 31(2):639-47.

45. Dirican N, Karakaya Y, Gunes S, Daloglu F, Dirican A. Association of intratumoral tumor infiltrating lymphocytes and neutrophil-to-lymphocyte ratio are an independent prognostic factor in non-small cell lung cancer. Clin Respir J (2015). doi:10.1111/crj.12417

46. Blum KS, Pabst R. Lymphocyte numbers and subsets in the human blood. Do they mirror the situation in all organs? Immunol Lett (2007) 108(1):45-51. doi:10.1016/j.imlet.2006.10.009

47. Ross R. Inflammation or atherogenesis. N Engl J Med (1999) 340(2):115-26.

48. Proctor MJ, McMillan DC, Horgan PG, Fletcher CD, Talwar D, Morrison DS. Systemic inflammation predicts all-cause mortality: a Glasgow Inflammation Outcome Study. PLoS One (2015) 10(3):e0116206. doi:10.1371/journal. pone.0116206 
49. Verdoia M, Barbieri L, Di Giovine G, Marino P, Suryapranata H, De Luca G, et al. Neutrophil to lymphocyte ratio and the extent of coronary artery disease: results from a large cohort study. Angiology (2016) 67(1):75-82. doi:10.1177/0003319715577529

50. Patnaik JL, Byers T, DiGuiseppi C, Dabelea D, Denberg TD. Cardiovascular disease competes with breast cancer as the leading cause of death for older females diagnosed with breast cancer: a retrospective cohort study. Breast Cancer Res (2011) 13(3):R64. doi:10.1186/bcr2901

51. Schairer C, Mink PJ, Carroll L, Devesa SS. Probabilities of death from breast cancer and other causes among female breast cancer patients. J Natl Cancer Inst (2004) 96(17):1311-21. doi:10.1093/jnci/djh253

52. Núñez J, Núñez E, Bodí V, Sanchis J, Miñana G, Mainar L, et al. Usefulness of the neutrophil to lymphocyte ratio in predicting long-term mortality in ST segment elevation myocardial infarction. Am J Cardiol (2008) 101(6):747-52. doi:10.1016/j.amjcard.2007.11.004

53. Tamhane UU, Aneja S, Montgomery D, Rogers EK, Eagle KA, Gurm HS. Association between admission neutrophil to lymphocyte ratio and outcomes in patients with acute coronary syndrome. Am J Cardiol (2008) 102(6):653-7. doi:10.1016/j.amjcard.2008.05.006
54. Azab B, Zaher M, Weiserbs KF, Torbey E, Lacossiere K, Gaddam S, et al. Usefulness of neutrophil to lymphocyte ratio in predicting short- and longterm mortality after non-ST-elevation myocardial infarction. Am J Cardiol (2010) 106(4):470-6. doi:10.1016/j.amjcard.2010.03.062

55. Uthamalingam S, Patvardhan EA, Subramanian S, Ahmed W, Martin W, Daley $\mathrm{M}$, et al. Utility of the neutrophil to lymphocyte ratio in predicting longterm outcomes in acute decompensated heart failure. Am J Cardiol (2011) 107(3):433-8. doi:10.1016/j.amjcard.2010.09.039

Conflict of Interest Statement: The authors declare that this research was conducted in the absence of any commercial or financial relationships that could be construed as a potential conflict of interest.

Copyright (c) 2016 Rimando, Campbell, Kim, Tang and Kim. This is an open-access article distributed under the terms of the Creative Commons Attribution License (CC $B Y)$. The use, distribution or reproduction in other forums is permitted, provided the original author(s) or licensor are credited and that the original publication in this journal is cited, in accordance with accepted academic practice. No use, distribution or reproduction is permitted which does not comply with these terms. 